\title{
Advances in pervasive health
}

\author{
Michael J. O'Grady • Brian Caulfield • \\ Gregory M. P. O'Hare
}

Published online: 10 July 2012

(C) Springer-Verlag 2012

Pervasive Health seeks to harness developments in pervasive computing technologies and harness them in the health domain. This domain may be interpreted in its widest possible sense, including the medical aspect both physical and mental, but also that of care management, education, community and occupational health. Such is the potential of pervasive computing technologies that the domain of pervasive health itself may ultimately fracture into a number of specialised domains, as the potential of the technology is being increasingly realised in practice. Indeed, this is already occurring with increased research activity being directed towards Ambient Assisted Living (AAL) in response to ongoing societal aging patterns.

A number of key challenges still remain which Pervasive Health must seek to address. From the technological perspective, one such challenge is to realise solutions that are:

1. Ambient: The need for technologies which are sufficient for the delivery of the required functionality but which are non-obtrusive. Such technological solutions need to capable of being subsumed into the fabric of our everyday lives;

2. Durable: The durability of such technologies must embrace the everyday lives of the subjects who's needs they serve. Often such technology will be subjected to

\footnotetext{
M. J. O’Grady $(\bowtie) \cdot$ B. Caulfield · G. M. P. O'Hare

Centre for Sensor Web Technologies, University College

Dublin, Belfield, Dublin 4, Ireland

e-mail: Michael.j.ogrady@ucd.ie

B. Caulfield

e-mail: b.caulfield@ucd.ie

G. M. P. O'Hare

e-mail: Gregory.OHare@ucd.ie
}

the relentless trials of our everyday lives with adverse weather conditions, knocks and impacts offering some illustration as to the treatments that await;

3. Power-aware: many wearable technologies must operate for prolonged periods of time while powered from resource constrained power sources like batteries. In order to ensure longevity of operation such systems must be power aware offering the possible of degradation of service and/or power scavenging possibilities;

4. Always-on: Pervasive Health applications by their very nature are intolerant of system outages and generally demand a near $24 / 7$ service. This is a challenge where one must deal with the imponderables of lossy and incomplete wireless network coverage, failure prone individual devices, and energy depletion. Such systems must incorporate system redundancies so as to mitigate against such issues;

5. Intelligent: Solutions must be capable of evolving to reflect the dynamics of a given condition or indeed the changing needs or circumstances of a given individual. A deployed solution must exhibit sufficient adaptivity so as to grow with the needs of the individual. Such adaptivity demands inherent system intelligence;

6. Accurate: Where health is being monitored the inherent accuracy of data captured is fundamentally important. As such, safeguards must be included to ensure the provenance of the data and the (re)calibration of sensing devices. Where Quality of Service thresholds have been established then these must be ensured and enforced;

7. Price Sensitive: The solutions must be sensitive to price thus ensuring access to and take-up of those solutions developed by a sufficiently large percentage of the population at large;. 
The aim of this Special Issue (SI) is to present a spectrum of ongoing developments in Pervasive Health. Papers for the SI were selected from those presented at the 5th International Conference on Pervasive Computing Technologies for Healthcare (PervasiveHealth 2011), in Dublin, Ireland between the 23rd and 26th of May 2011. Seven papers were identified, based on feedback from the reviewers on the Programme Committee. Authors were invited to submit extended and revised versions of their papers. Each paper was then subjected to a second review, using a different group of reviewers, and each was then revised to ensure quality. The scope of the papers elected for inclusion indicate the broad applicability and potential of pervasive health technologies.

Lanatà et al. describe HATCAM, a wireless and wearable eye-gaze tracking system. Determining emotional state is important in many diagnostic cases, but especially perhaps in cases where mental health is an issue. Thus a robust and inexpensive method of eye tracking may prove indispensible in many instances as a remote diagnostic aid.

Ye et al. describe a uniform approach towards working with smart home datasets. Given the time and effort that must be extended in constructing and validating a arbitrary dataset, it is of most importance that maximum usage be gleaned from each instance. In enabling a standardised framework for dataset processing, it is hoped that new datasets may be made available, and critical issues such as data fusion and benchmarking can be addressed more thoroughly.

Mooney et al. consider how Volunteered Geographic Information (VGI) may be harnessed in pervasive health applications. VGI may be regarded as an example of crowd-sourcing, a mechanism for harnessing the power of crowds to construct datasets. Though successfully demonstrated in the environmental monitoring sphere, there is no reason why the same principles cannot be harnessed to compile health-related datasets in a variety of areas.

Vathsangam et al. consider the case of calorie or energy consumption across a population. A traditional approach tends to use generic population models; however, the issue of developing user-specific models is appealing for many reasons. A hierarchical model is presented, based on inertial sensors, that measures energy expenditure across a target population.

Frenken et al. propose a novel approach for mobility analysis using ambient sensor technologies. This has been compared with a conventional approach and initial feedback is positive. Such an approach offers interesting possibilities for remote mobility analysis in domestic situations.

Chaudry et al. consider how low-literacy populations estimate portion size. This is a very practical issue, and has applications in food and medicine intake. A number of guidelines are presented for designing interfaces that aid such populations monitor their fluid intake.

Chamadiya et al. focus on the clinical environment and how ECG monitoring may be achieved in a contactless manner. Such a development has significant implications for how vital signs may be monitored continuously in nonICU settings.

The guest editors would like to formally thank all the authors for submitting their papers. The efforts of the reviewers are also gratefully acknowledged. Finally, the encouragement of the Editor-in-Chief of this journal, Prof. Vincenzo Loia, in making this SI happen, is also acknowledged. 